\title{
Characterization of Polylactide-Stabilized Gold Nanoparticles and Its Application in the Fabrication of Electrochemical DNA Biosensors
}

\author{
Noordiana Nordin, ${ }^{a}$ Nor Azah Yusof, ${ }^{* a, b}$ Jaafar Abdullah, ${ }^{a, b}$ Son Radu ${ }^{c}$ and Reza Hajian $* a$ \\ ${ }^{a}$ Institute of Advanced Technology, ${ }^{b}$ Department of Chemistry, Faculty of Science and ${ }^{c}$ Food Safety \\ Research Centre, Faculty of Food Science and Technology, Universiti Putra Malaysia, 43400 UPM \\ Serdang, Selangor, Malaysia
}

\begin{abstract}
In this work, two different approaches to gold nanoparticles (AuNPs) have been explored for the modification of screen-printed electrode based on AuNPs and AuNPs stabilized with polylactic acid (PLA). The modified substrate has been characterized using UV-Vis spectroscopy, X-ray diffraction (XRD), transmission electron microscopy (TEM), field emission scanning electron microscopy (FESEM) and cyclic voltammetry. Both synthesized AuNPs were studied in terms of stability, sensitivity and reproducibility to enhance the sensing capability of modified electrodes. The PLA-stabilized AuNPs form strong structured nanoparticles and stabilize in aqueous solution. A larger active surface area $\left(0.41 \mathrm{~cm}^{2}\right)$ and lower charge transfer resistance $\left(\mathrm{R}_{\mathrm{ct}}\right)$ value were reported in the modification of sensing material with PLA-stabilized AuNPs, which resulted in enhancement of sensitivity. Therefore, AuNPs in PLA can be used as a potential alternative modifier for sensing chemicals and biomolecules in electrochemical sensors.
\end{abstract}

Keywords: electrochemical DNA biosensor, screen-printed carbon electrode, polylactic acid, gold nanoparticles

\section{Introduction}

Research in the development of deoxyribonucleic acid (DNA) biosensor based on the hybridization process has enormously increased in recent years due to its importance towards various applications including environmental monitoring, ${ }^{1,2}$ clinical diagnosis, ${ }^{3,4}$ gene analysis, ${ }^{5,6}$ and food safety. ${ }^{7}$ A lot of methods have been explored to determine DNA such as fluorometry, ${ }^{8}$ chromatography, ${ }^{9}$ spectrophotometry, ${ }^{10}$ chemiluminescence ${ }^{11}$ and electrochemical methods. ${ }^{12}$ Electrochemical methods gained much attention among other methods due to their high sensitivity, low cost instrumentation, fast response, simplicity and portability in detection. ${ }^{13}$

The sensitivity of electrochemical DNA sensors highly depends on the signal amplification; thus, suitable sensing materials must be carefully applied. ${ }^{14}$ Unique properties of nanomaterials have contributed to unlimited options of sensing materials. Interestingly, gold nanoparticles (AuNPs), as one of the widely used nanomaterials, differ from their bulk counterparts, and have various interesting spectroscopic, electronic and chemical properties, including

*e-mail: azahy@upm.edu.my,rezahajian@upm.edu.my their high surface-to-volume ratio, nanometer size and ability to couple with electromagnetic wave or with surface plasmon of neighboring metal particles. ${ }^{15}$ Hence, they offer an open biological assembly platform to enhance the electron-transfer process between biological molecules and substrate. ${ }^{16}$ Organic linker molecules such as thiols and polymers are commonly used to fabricate AuNPs-modified electrodes by assembling AuNPs on electrode surfaces.

Polylactic acid (PLA) is one of the biodegradable polymers that have recently gained attention in bioanalysis areas due to their special characteristics including subcellular size, biocompatibility as well as controlled and sustained release properties. ${ }^{17,18}$ Highly stable PLA-AuNPs have been proven to significantly improve both surface area and particle size of modified electrodes. ${ }^{19}$ Earlier research has reported that stabilization of AuNPs takes place between the carboxylic groups of polymer matrix. ${ }^{20}$ Practically, the application of screen-printed carbon electrode (SPCE) as a disposable sensor for detection of DNA hybridization is attractive since it allows low-cost electrochemical biosensor. PLA-stabilized AuNPs modified electrodes have been described previously as successful and practical sensor identifying As ${ }^{\mathrm{III}}$ and sulfide in water. ${ }^{21,22}$ Although the same electrode modification was extended 
in our study, we used a different approach for preparation of PLA-stabilized AuNPs-based electrochemical sensor. To the best of our knowledge, there is no record on comparative study between AuNPs and PLA-stabilized AuNPs, and their properties as modified electrodes. Therefore, this work reports the characteristics of AuNPs and PLA-stabilized AuNPs as a modified substrate for construction of electrochemical sensors. Properties of modified electrode and its preliminary application in DNA biosensors were also described in detail.

\section{Experimental}

\section{Materials and instruments}

PLA with the molecular weight of 320,000 Da was used for stabilization of AuNPs. $\mathrm{CHCl}_{3}$ was purchased from Merck and used as a solvent. $\mathrm{HAuCl}_{4} \cdot 3 \mathrm{H}_{2} \mathrm{O}$ and sodium citrate were purchased from Sigma-Aldrich. All reagents were of analytical grade and used without further purification. $\mu$ Autolab III voltammetric analyzer (Eco-Chemie) incorporated with general purpose electrochemical system (GPES) 4.9 software was used to analyze samples. The characterization of AuNPs was studied using UV-Vis spectrophotometer (Shimadzu UV-Vis-1650), X-ray diffraction (XRD) (Philips PW 3040/60 MPD X'PERT Pro Analytical), transmission electron microscopy (TEM) (Hitachi H-7100) and field-emission scanning electron microscopy (FESEM) with energy dispersive X-ray (EDX) analyzer (JEOL JSM 7600F).

\section{Preparation and characterization of AuNPs}

AuNPs were synthesized according to the method reported by Nasir and Nur. ${ }^{23}$ Briefly, $10 \mathrm{~mL}$ of sodium citrate solution $\left(38.8 \mathrm{mmol} \mathrm{L}^{-1}\right)$ were added to $100 \mathrm{~mL}$ of aqueous chloroauric solution $\left(1 \mathrm{mmol} \mathrm{L}^{-1}\right)$ under boiling. The color of solution first changed from yellow to blackish and finally to dark ruby red after about $10 \mathrm{~min}$. The solution was then cooled to ambient temperature and kept in fridge.

\section{Preparation and characterization of PLA-stabilized AuNPs}

Prior to preparation of PLA sheet, PLA pellets were placed in stainless steel mould, preheated for melting at $190{ }^{\circ} \mathrm{C}$ for $10 \mathrm{~min}$ and then pressed under pressure of 2.2 MPa for $1 \mathrm{~min}$. The melted PLA was cooled and ready for usage. About $0.68 \mathrm{mg}$ of PLA sheets were cut into small pieces, dissolved in $5 \mathrm{~mL}$ of chloroform and stirred vigorously in room temperature, and then $10 \mathrm{~mL}$ of AuNPs solution were added to the dissolved PLA. The mixture was stirred in room temperature until completely homogeneous. The characterization of PLA-stabilized AuNPs was determined with the same instruments as described in AuNPs characterization.

\section{Preliminary study of biosensor fabrication}

A volume of $25 \mu \mathrm{L}$ of homogenous AuNPs and PLA-stabilized AuNPs solution was pipetted on SPCE and dried at ambient temperature for $24 \mathrm{~h}$ prior to use. For preliminary study, the effect of six different types of supporting electrolytes at $0.1 \mathrm{~mol} \mathrm{~L}^{-1}$ including phosphate buffer, potassium chloride, potassium dihydrogen phosphate $\left(\mathrm{KH}_{2} \mathrm{PO}_{4}\right)$, phosphate-buffered saline (PBS), Tris-EDTA and Tris- $\mathrm{HCl}$ were studied on the sensitivity of electrochemical biosensor. The effect of $\mathrm{pH}$ was studied in the range of 2 to 12 . All experiments were recorded by cyclic voltammetry $(\mathrm{CV})$ in the potential range of -0.8 to $0.3 \mathrm{~V}$ for 3 cycles. The performance of the developed biosensor was investigated by $\mathrm{CV}$ analysis in the presence of $20 \mu \mathrm{mol} \mathrm{L}-1$ methylene blue (MB).

\section{Results and Discussions}

\section{Characterization of AuNPs and PLA-stabilized AuNPs}

The formation of AuNPs was indicated by the color change of aqueous solution from light yellow to dark ruby red in the presence of sodium citrate. According to Supplementary Information Figure S1, absorption peaks of AuNPs and PLA-stabilized AuNPs solutions are in the wavelength range of 520 to $540 \mathrm{~nm}$, showing the presence of AuNPs in the solution. The absorbance of AuNPs and PLA-stabilized AuNPs appears at wavelengths ranging from 500 to $600 \mathrm{~nm}$ depending on their particle size. ${ }^{24}$ Figure 1 shows XRD pattern of synthesized AuNPs and PLA-stabilized AuNPs. The strong diffraction peaks with $2 \theta$ values located at $38.2,44.4,64.7$ and $77.7^{\circ}$ correspond to Bragg's reflection from (111), (200), (220) and (311) planes of the face-centered cubic (fcc) metal gold nanostructures. These results correspond with previous reports on

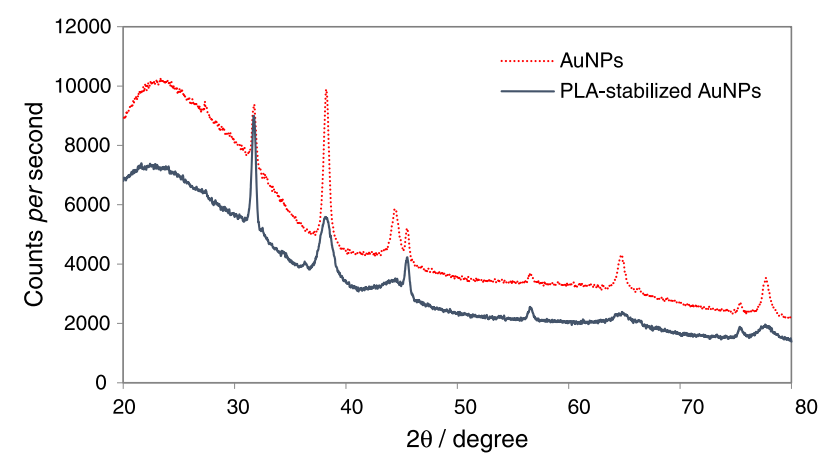

Figure 1. X-Ray diffraction spectra of AuNPs and PLA-stabilized AuNPs. 
AuNPs by Rastogi and Arunachalam. ${ }^{25}$ The broadening of XRD peaks were indicated on formation of nanosized PLA-stabilized AuNPs.

Furthermore, all diffraction peaks of AuNPs appeared on the PLA-stabilized AuNPs indicating that the structure of AuNPs is stable in the presence of polylactide. Figure 1 shows some new diffraction peaks for PLA-stabilized AuNPs at $2 \theta$ regions of $32^{\circ}$, which indirectly indicate the good crystallization of polymers (PLA) on the surface of AuNPs. The crystalline sizes of AuNPs in Table 1 were determined from (111) plane by the application of Scherrer's equation as:

$\mathrm{L}=\mathrm{k} \lambda / \beta \cos \theta$

where $\mathrm{L}$ is the crystallites mean dimension, $\mathrm{k}$ is a constant equal to $0.94, \lambda$ is $\mathrm{Cu} \mathrm{K} \alpha$ radiation wavelength $(0.154 \mathrm{~nm})$, $\beta$ is the full width at half maximum (FWHM) of diffraction peak and $\theta$ is the diffraction angle. The calculated crystalline sizes of AuNPs are 32 and $27 \mathrm{~nm}$ for AuNPs and PLA-stabilized AuNPs samples, respectively. It was observed that FWHM is inversely proportional to the crystalline size. The smaller size of PLA-stabilized AuNPs might be due to the compression of AuNPs by a fairly thin PLA layer on the surface. These results clearly indicate that the PLA-stabilized AuNPs form strong structured nanoparticles and stabilize in aqueous solution.

The size and morphology of AuNPs and PLA-stabilized AuNPs were further studied by TEM. The TEM images and particle size frequency distribution curve of AuNPs and PLA-stabilized AuNPs are presented in Figure 2, which exhibit spherical and oval shapes. The shapes displayed in Figure 2a are largely spherical and highly dispersed as compared to those in Figure 2b. The nanostructure shapes of Figure $2 \mathrm{~b}$ are clearly outlined, although the shapes are widely agglomerated. However, these results disagreed with the crystallite sizes found in the XRD results, as they are an estimate value calculated using Scherrer's equation, whereas TEM directly measures the actual particle size. Nanda et $a l .{ }^{26}$ found that the calculated particle size by TEM technique has larger size due to large amount of several crystalline domains oriented in different directions inside a single crystallite. Hence, the sensitivity of XRD to the dimensions of such individual local crystalline domains within a single nanocrystallite projected smaller particle size. In line with that, Rath et al. ${ }^{27}$ also found the smaller size estimated in XRD as compared to TEM, which indicated that particles were polycrystalline as they contained an abundance of smaller crystallites (subparticles) inside them. In addition, Corbierre et al..$^{28}$ discovered that polymer-stabilized gold nanoparticles have larger diameters and polydispersities.

Figure 3 shows FESEM images and EDX spectra of SPCE surface (5 $\mathrm{mm}$ diameter) before and after modification with AuNPs and PLA-stabilized AuNPs. It is clear that the modification of SPCE with PLA-stabilized AuNPs has greater density and weight percentage as well as larger surface coverage of nanoparticles.

\section{Electrochemical characterization of PLA-stabilized AuNPs modified SPCE}

The electrochemical performance of modified electrode was evaluated using potassium ferrocyanide solution by $\mathrm{CV}$. Due to the fast electrochemical reaction and fine reversibility of ferrocyanide ion $\left(\left[\mathrm{Fe}(\mathrm{CN})_{6}\right]^{3-/ 4-}\right)$, it has been used for electrochemical study of SPCE after modification with AuNPs in aqueous and organic buffer solutions.

Supporting electrolyte has important role in electrochemical biosensors as it affects the activation of DNA in aqueous solution without interrupting DNA structure. Proper choice of buffer must be studied carefully as it is correlated with the rate of charge transfer. CV of $\left[\mathrm{Fe}(\mathrm{CN})_{6}\right]^{3-/ 4-}$ ion at modified SPCE surface was significantly studied at different kinds of supporting electrolytes. Based on Figure S2, PBS $\left(0.1 \mathrm{~mol} \mathrm{~L}^{-1}\right)$ has higher sensitivity and was selected as working electrolyte for DNA sensors based on PLA-stabilized AuNPs. PBS is stable at various temperatures, is non-toxic to DNA, has microbial resistance and can be stored for several weeks.

$\mathrm{pH}$ of solution plays an important role in providing appropriate environment for DNA to operate efficiently. However, DNA molecules may denature at high $\mathrm{pH}$ values. From the results shown in Figure S3, by increasing pH from 2.0 to 7.0, the anodic peak current of $\left[\mathrm{Fe}(\mathrm{CN})_{6}\right]^{3-14}$ ion was increased and reached the highest at $\mathrm{pH} 7.0$, so it was chosen as the optimum working $\mathrm{pH}$ for PLA-stabilized AuNPs modified DNA sensors.

Table 1. Crystal size of AuNPs and PLA-stabilized AuNPs

\begin{tabular}{|c|c|c|c|c|}
\hline Sample & $2 \theta /$ degree & FWHM / $2 \theta$ & d-Spacing / $\AA$ & Crystal size / nm \\
\hline AuNPs & 38.2037 & 0.2755 & 2.3558 & 32 \\
\hline PLA-stabilized AuNPs & 31.6820 & 0.3149 & 2.8243 & 27 \\
\hline
\end{tabular}

FWHM: full width at half maximum; AuNPS: gold nanoparticles; PLA: polylactic acid. 

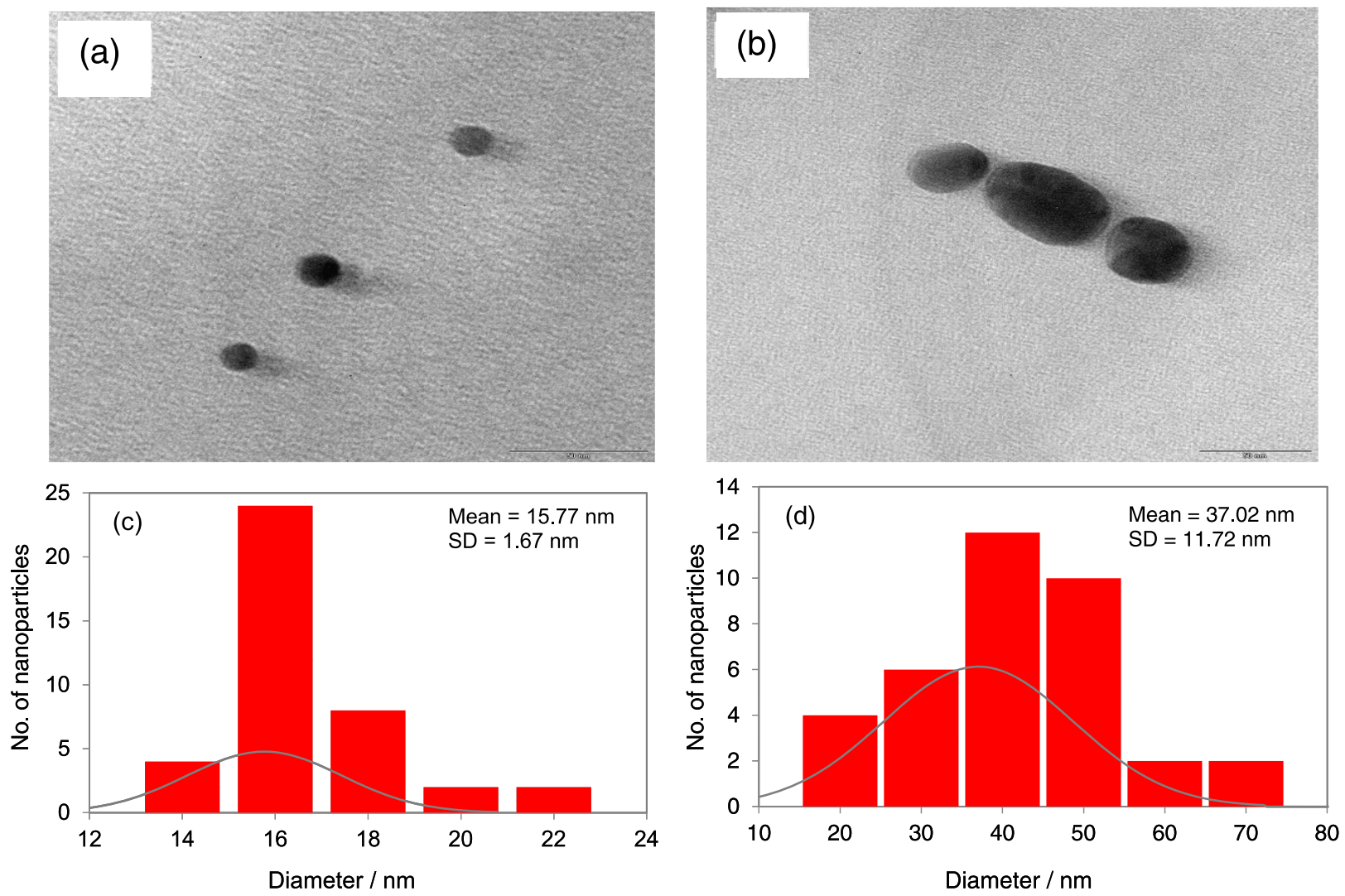

Figure 2. TEM images of (a) AuNPs and (b) PLA-stabilized AuNPs. Frequency diameter distributions for (c) AuNPs and (d) PLA-stabilized AuNPs. SD: standard deviation.

\section{Effect of scan rate}

Based on the CV obtained (Figure 4), the cathodic peak current of $\left[\mathrm{Fe}(\mathrm{CN})_{6}\right]^{3-4-}$ ion significantly increased in the scan rate of 0.01 to $1.0 \mathrm{~V} \mathrm{~s}^{-1}$. Lower scan rate shows more reversible voltammograms with smaller charging current. Hence, scan rate of $0.1 \mathrm{~V} \mathrm{~s}^{-1}$ was chosen to be applied in the electrochemical study as the electrochemical reaction was reversible.

\section{Properties of modified electrode}

The property of PLA-stabilized AuNPs modifier was assessed by calculating the active surface area using Randles-Sevcik equation as:

$\mathrm{i}_{\mathrm{pc}}=\left(2.69 \times 10^{5}\right) \mathrm{n}^{3 / 2} \mathrm{AD}^{1 / 2} \mathrm{Cv}^{1 / 2}$

where $i_{p c}$ is the cathodic peak current, $n$ is the number of electrons participating in the redox reaction, $\mathrm{A}$ is the activate surface area of electrode $\left(\mathrm{cm}^{2}\right), \mathrm{D}$ is diffusion coefficient of $\mathrm{K}_{3} \mathrm{Fe}(\mathrm{CN})_{6}$ in solution $\left(7.6 \times 10^{-6} \mathrm{~cm}^{2} \mathrm{~s}^{-1}\right)$, $\mathrm{C}$ is the concentration of $\mathrm{K}_{3} \mathrm{Fe}(\mathrm{CN})_{6}\left(\mathrm{~mol} \mathrm{~L}^{-1}\right)$ and $\mathrm{v}$ is scan rate $\left(\mathrm{V} \mathrm{s}^{-1}\right)$. The active surface area was calculated as 0.26 and $0.31 \mathrm{~cm}^{2}$ for bare SPCE and SPCE/AuNPs, respectively, while it was $0.41 \mathrm{~cm}^{2}$ after modification with PLA-stabilized AuNPs. In fact, the active surface area for modified PLA-stabilized AuNPs electrode was higher (Figure 5). This result implies that the application of PLA-stabilized AuNPs as electrochemical sensing material can improve the active surface area better than AuNPs. This result is consistent with previous work of polymer-embedded gold nanoparticles, which demonstrates that higher active surface area showed better electron-transfer process, resulting in higher sensitivity. ${ }^{29}$ The electrochemical reaction of modified SPCE is under diffusion control process, which was indicated by the dependence of reduction peak current of $\mathrm{K}_{3}\left[\mathrm{Fe}(\mathrm{CN})_{6}\right]^{3-/ 4-}$ on square root of scan rate $\left(v^{1 / 2}\right)$.

Electrochemical impedance spectroscopy (EIS) (Figure 6) shows that the charge transfer resistance value of the bare SPCE is $1932 \Omega$, while after modification with AuNPs (SPCE/AuNPs) and PLA-stabilized AuNPs, the resistance decreased to 1730 and $1444 \Omega$, respectively. The EIS results are in agreement with the CV measurements as shown in Figure S4, which clearly exhibits the increase of the peak currents after modification with AuNPs and PLA-stabilized AuNPs. This indicates that the higher 

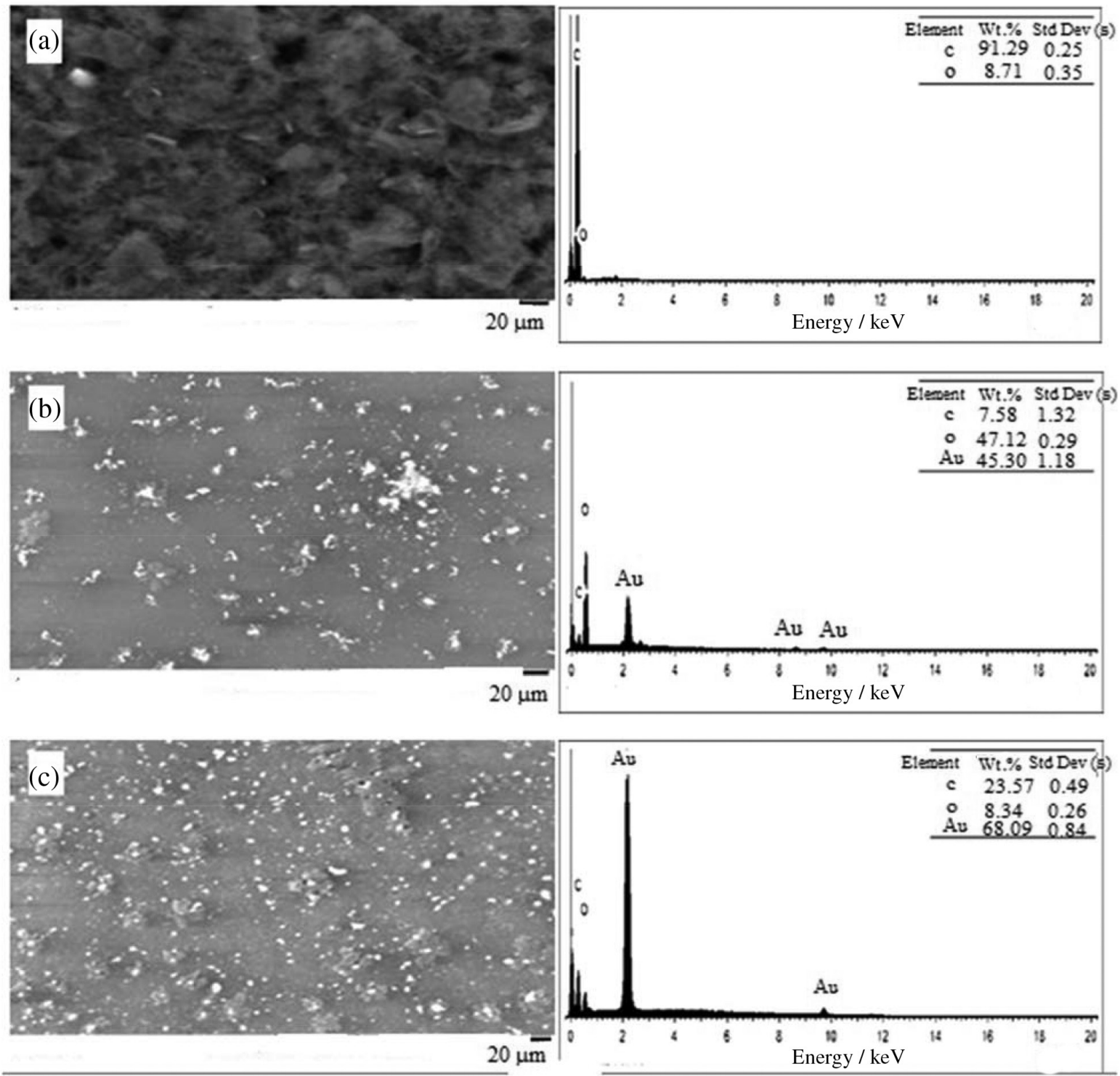

Figure 3. FESEM images and EDX spectra of (a) bare SPCE, (b) AuNPs modified SPCE and (c) PLA-stabilized AuNPs modified SPCE.

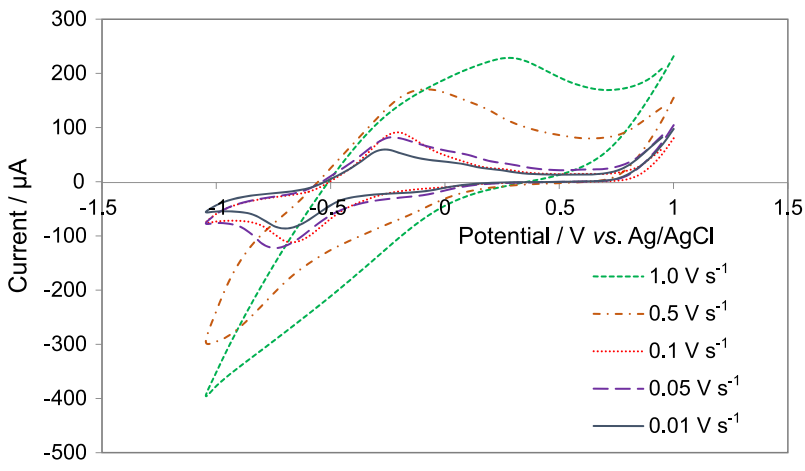

Figure 4. Cyclic voltammograms of $\left[\mathrm{Fe}(\mathrm{CN})_{6}\right]^{3-14}$ at different scan rates: $1.0,0.5,0.1,0.05$ and $0.01 \mathrm{~V} \mathrm{~s}^{-1}$ at potential range of 1 to $-1 \mathrm{~V}$.

sensitivity is inversely proportional to the lower charge transfer resistance. ${ }^{30}$

The repeatability of constructed electrodes was investigated by repetitive recording of $\mathrm{CV}$ of $\mathrm{K}_{3}\left[\mathrm{Fe}(\mathrm{CN})_{6}\right]^{3-14-}$ for six cycles. The relative standard deviations (RSD, \%) were determined as 4.26 and $9.97 \%$ for SPCE/PLA-stabilized AuNPs and SPCE/AuNPs, respectively, showing that the fabricated electrochemical sensor based on PLA-stabilized gold nanoparticles is more reusable and can be used for several times. Moreover, six modified electrodes were fabricated independently with the same procedure, which gave a satisfactory RSD value of $4.05 \%$ on the oxidation peak current of $\mathrm{K}_{3}\left[\mathrm{Fe}(\mathrm{CN})_{6}\right]^{3-} /^{4-}$, while it was $9.99 \%$ for SPCE/AuNPs, showing better reproducibility for PLA-stabilized AuNPs (Table 2). The modified substrate based on PLA-AuNPs can be stored at room temperature for a long time and can be used for recording $20 \mathrm{CV}$ with $18 \%$ signal reduction while SPCE/AuNPs substrate has lower stability ( $38 \%$ reduction in peak current after 20 cycles). 

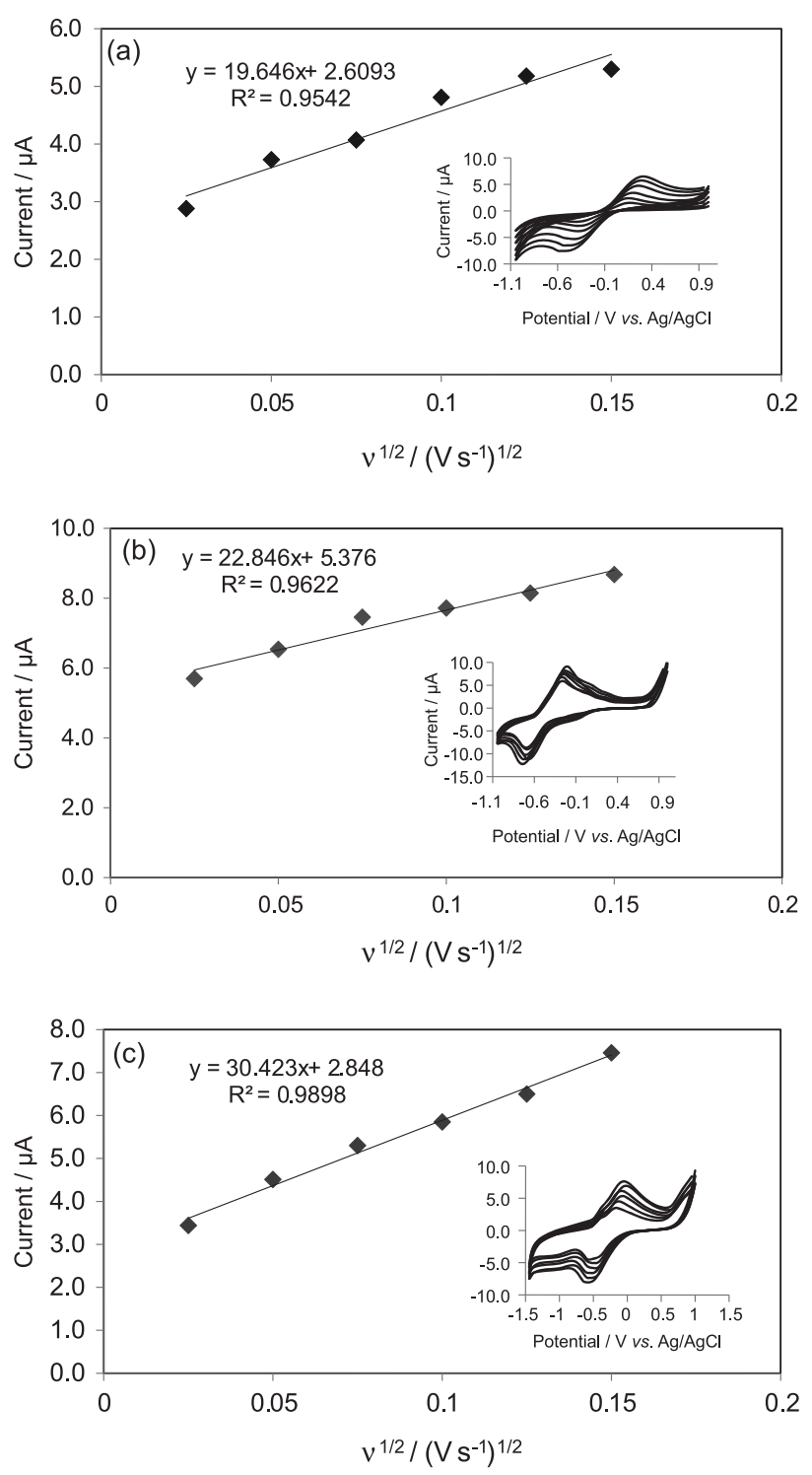

Figure 5. Cyclic voltammograms and plot of oxidation peak currents against square root of scan rate for (a) bare SPCE, (b) SPCE/AuNPs and (c) SPCE/PLA-stabilized AuNPs in $1.0 \mathrm{mmol} \mathrm{L}^{-1} \mathrm{~K}_{3}\left[\mathrm{Fe}(\mathrm{CN})_{6}\right]$. The scan rates were $50,100,150,200,250,300$ and $350 \mathrm{mV} \mathrm{s}^{-1}$.

Fabrication of electrochemical DNA biosensors based on PLA-AuNPs and AuNPs modified SPCE

Figure 7 shows the fabrication process of PLA-AuNPs and AuNPs modified SPCE. The working electrode substrate on SPCE was drop cast with $25 \mu \mathrm{L}$ of homogenous AuNPs

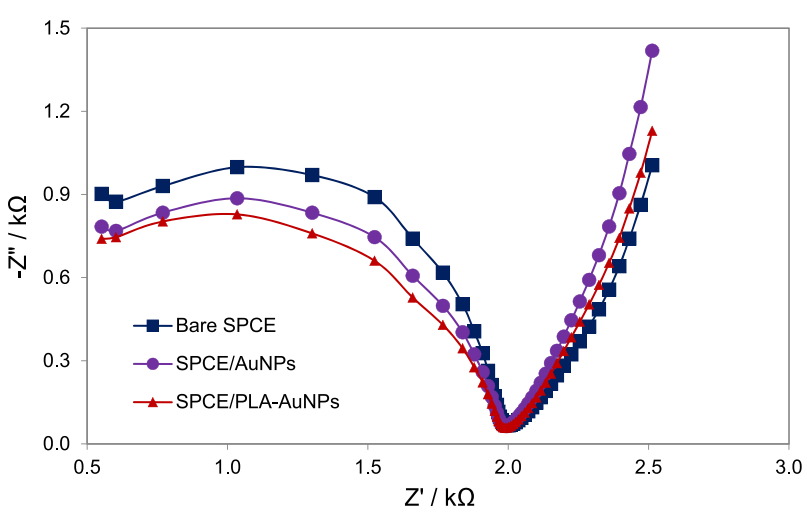

Figure 6. EIS study of modified electrodes in $1.0 \mathrm{mmol} \mathrm{L}^{-1} \mathrm{~K}_{3}\left[\mathrm{Fe}(\mathrm{CN})_{6}\right]$. Frequency range: $0.1-10^{5} \mathrm{~Hz}$, amplitude: $5 \mathrm{mV}$.

and PLA-stabilized AuNPs solution, then dried in ambient temperature for $24 \mathrm{~h}$. The modified SPCE was denoted as AuNPs/SPCE and PLA-AuNPs/SPCE. Self-assembled monolayer technique based on thiol-AuNPs bonding process was used for bonding probe single-stranded DNA (ssDNA) on the surface of AuNPs and PLA-AuNPs modified electrode by bonding AuNPs to thiol groups. For hybridization process, the complementary target DNA was introduced into the probe DNA-AuNPs/SPCE and DNA/PLA-AuNPs/SPCE that converted into electrochemical signal using $\mathrm{MB}$ as a redox indicator, which was able to incorporate at DNA surface. The performance of fabricated biosensor was evaluated using PBS ( $\mathrm{pH}$ 6.8) at $100 \mathrm{mV} \mathrm{s}^{-1}$ by CV after $30 \mathrm{~min}$ of incubation time for MB.

In this study, performance of the developed biosensor was further investigated in the presence of $\mathrm{MB}$ as a redox label, which is widely known to have an affinity towards DNA. In general, the degree of MB immersion time was studied in the range of 1.0 to $180 \mathrm{~min}$. The immersion time of 30 min was chosen as the optimum duration to enhance sensitivity. There are two possible mechanisms of $\mathrm{MB}$ interaction, namely with an interaction between guanine bases which are mainly in ssDNA, or by an electrostatic interaction with the negatively charged phosphate groups. ${ }^{31}$ The advantage of MB utilization in the electrochemical reaction happens at comparatively low potential where the interferences from other electroactive species and background can be minimized compared to detection with label-free electrochemical method.

Table 2. Characteristics of modified electrodes based on AuNPs and PLA-stabilized AuNPs

\begin{tabular}{|c|c|c|c|}
\hline \multirow{2}{*}{ Modified electrode } & \multicolumn{2}{|c|}{$\mathrm{RSD} / \%$} & \multirow{2}{*}{ Signal reduction $/ \%$} \\
\hline & Repeatability & Reproducibility & \\
\hline SPCE/PLA-stabilized AuNPs & 4.26 & 4.05 & 18 \\
\hline SPCE/AuNPs & 9.97 & 9.99 & 38 \\
\hline
\end{tabular}

RSD: relative standard deviation; SPCE: screen-printed carbon electrode; PLA: polylactic acid; AuNPs: gold nanoparticles. 


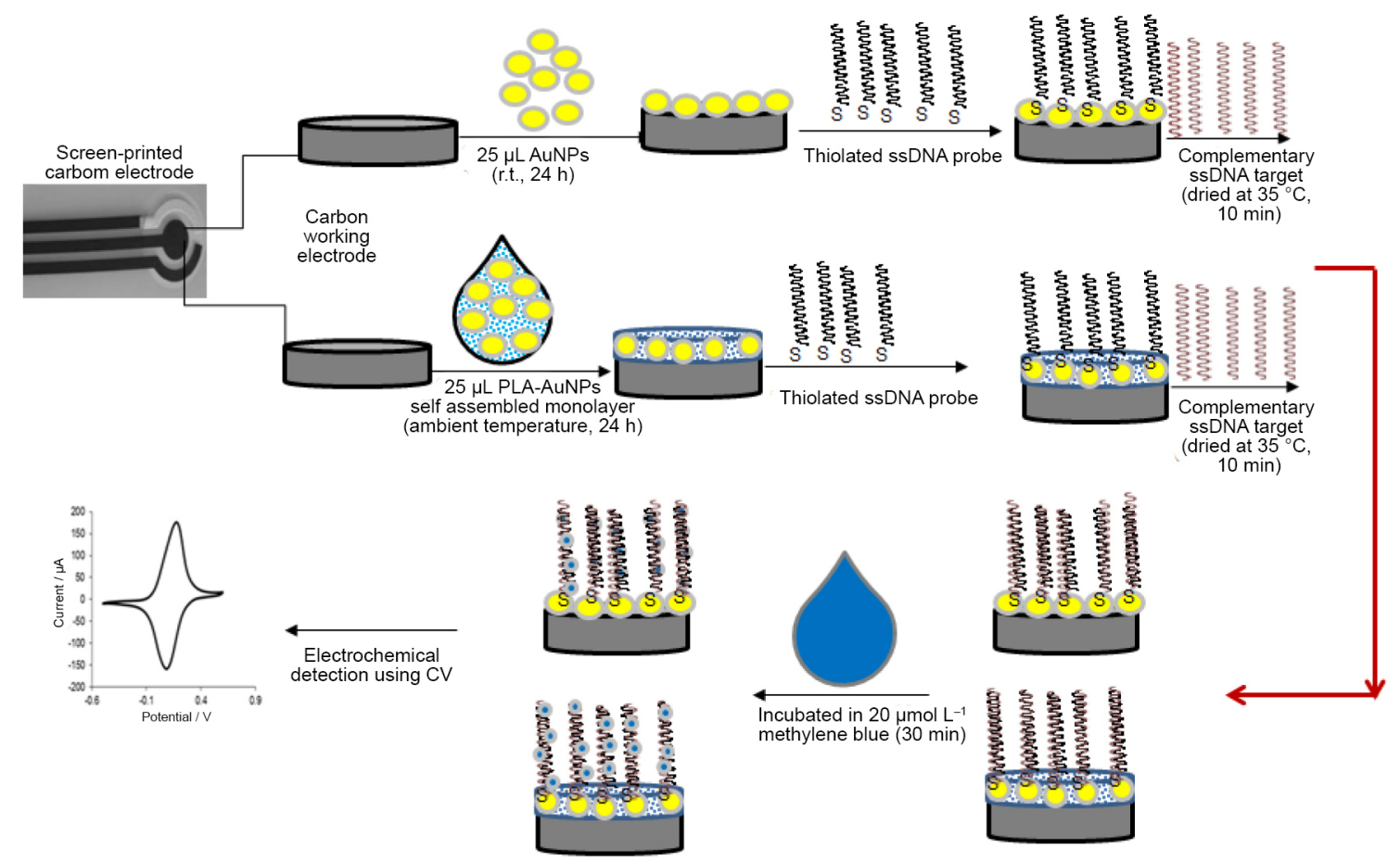

Figure 7. Fabrication process of PLA-AuNPs and AuNPs modified screen-printed carbon electrode for electrochemical biosensor.

Figure 8 indicates representative $\mathrm{CV}$ peak current obtained at PLA-AuNPs and AuNPs modified SPCE coated with the same probe DNA concentration and complementary target DNA. In order to get higher sensitivity by CV, SPCE was pre-treated using $0.5 \mathrm{~mol} \mathrm{~L}^{-1}$ acetate buffer solution

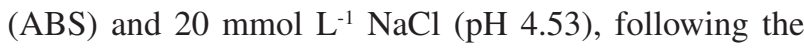
modified method from Issa et al..$^{32}$ prior to measurement throughout the experiment. An increment in MB peak current happened after immobilization of thiolated single stranded DNA onto PLA-AuNPs and AuNPs modified electrode. Nevertheless, the peak current of MB significantly decreased about 1-fold lower upon hybridization with target DNA. This finding is in correlation with Khezrian et al. ${ }^{33}$ and Mao et al. ${ }^{34}$ who also reported high MB accumulation

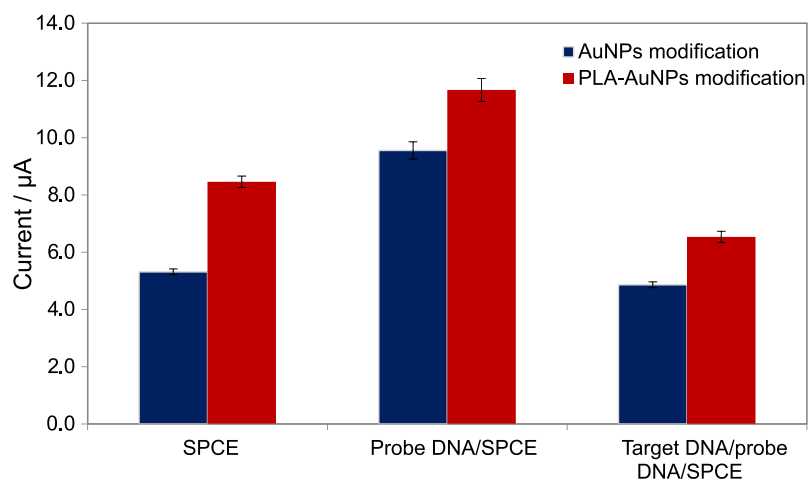

Figure 8. Peak current of DNA detection using fabricated biosensor after $30 \mathrm{~min}$ incubation in $20 \mu \mathrm{mol} \mathrm{L}-1 \mathrm{MB}$ in $0.1 \mathrm{~mol} \mathrm{~L}^{-1} \mathrm{PBS}$ (pH 6.8) at $100 \mathrm{mV} \mathrm{s}^{-1}$. on double-stranded DNA surface. This result shows that the detection of target DNA by the fabricated electrochemical DNA biosensor was enabled by MB utilization as redox indicator. The electrode modification with PLA-stabilized AuNPs shows higher sensitivity and stability in comparison with AuNPs alone on the surface of SPCE.

\section{Conclusions}

In this paper, two different gold nanoparticles were synthesized as modified substrates for using in electrochemical DNA biosensors. AuNPs stabilized with polylactic acid were found to be more stable and exhibited better characteristics as a modifier. The SPCE/PLA-stabilized AuNPs increases the sensitivity due to the larger active surface area produced and lower $\mathrm{R}_{\mathrm{ct}}$ value. The stability, reproducibility and repeatability of SPCE/PLA-stabilized AuNPs revealed satisfying results. The preliminary study shows that PLA-stablized gold nanoparticles is a reliable substrate for binding thiolated DNA oligumers in electrochemical DNA biosensors. Hence, the application of PLA-stabilized AuNPs has great potential to be applied as modifier for further sensor fabrication.

\section{Supplementary Information}

Supplementary information is available free of charge at http://jbcs.sbq.org.br as PDF file. 


\section{Acknowledgments}

The authors gratefully acknowledged the financial support of Universiti Putra Malaysia and all available facilities in the Department of Chemistry, Faculty of Science.

\section{References}

1. Xu, H. B.; Ye, R. F.; Yang, S. Y.; Li, R.; Yang, X.; Chin. Chem. Lett. 2014, 25, 29.

2. Li, M.; Gou, H.; Al Ogaidi, I.; Wu, N.; ACS Sustainable Chem. Eng. 2013, 1, 713.

3. Wang, Z.; Fan, Y.; Chen, J.; Guo, Y.; Wu, W.; He, Y.; Xu, L.; Fu, F.; Electrophoresis 2013, 34, 2177.

4. Hong, C. Y.; Chen, X.; Liu, T.; Li, J.; Yang, H. H.; Chen, J. H.; Chen, G. N.; Biosens. Bioelectron. 2013, 50, 132.

5. Zhao, H.; Lu, Z. H.; Chin. Chem. Lett. 2014, 25, 1559.

6. Luo, C.; Tang, H.; Cheng, W.; Yan, L.; Zhang, D.; Ju, H.; Ding, S.; Biosens. Bioelectron. 2013, 48, 132.

7. Vetrone, S. A.; Huarng, M. C.; Alocilja, E. C.; Sensors 2012, 12,10487

8. Bai, W.; Zheng, H.; Long, Y.; Mao, X.; Gao, M.; Zhang, L.; Anal. Sci. 2011, 27, 243.

9. Pereira, L. R.; Prazeres, D. M. F.; Mateus, M.; J. Sep. Sci. 2010, 33, 1175 .

10. Lin, H.; Li, M. X.; Liu, F.; Mihailovic, D.; Chin. Chem. Lett. 2014, 25, 645.

11. Luo, M.; Chen, X.; Zhou, G.; Xiang, X.; Chen, L.; Ji, X.; He, Z.; Chem. Commun. 2012, 48, 1126.

12. Lazerges, M.; Bedioui, F.; Anal. Bioanal. Chem. 2013, 405, 3705.

13. Liu, A.; Wang, K.; Weng, S.; Lei, Y.; Lin, L.; Chen, W.; Lin, X.; Chen, Y.; TrAC, Trends Anal. Chem. 2012, 37, 101.

14. Shi, A.; Wang, J.; Han, X.; Fang, X.; Zhang, Y.; Sens. Actuators, B 2014, 200, 206.

15. Daniel, M. C.; Astruc, D.; Chem. Rev. 2004, 104, 293.

16. Karhanek, M.; Kemp, J. T.; Pourmand, N.; Davis, R. W.; Webb, C. D.; Nano Lett. 2005, 5, 403.
17. Babu, A.; Templeton, A. K.; Munshi, A.; Ramesh, R.; J. Nanomater. 2013, 2013, 1.

18. Pridgen, E. M.; Langer, R.; Farokhzad, O. C.; Nanomedicine 2007, 2, 669 .

19. Chirea, M.; García-Morales, V.; Manzanares, J. A.; Pereira, C.; Gulaboski, R.; Silva, F.; J. Phys. Chem. B. 2005, 109, 21808.

20. Chen, H. Y.; Huang, B. H.; Lin, C. C.; Macromolecules 2005 , 38,5400 .

21. Song, Y. S.; Muthuraman, G.; Zen, J. M.; Electrochem. Commun. 2006, 8, 1369.

22. Song, Y. S.; Muthuraman, G.; Chen, Y.Z.; Lin, C. C.; Zen, J. M.; Electroanalysis 2006, 18, 1763.

23. Nasir, S.; Nur, H.; J. Fund. Sci. 2008, 4, 245.

24. Seoudi, R.; Fouda, A. A.; Elmenshawy, D. A.; Phys. B 2010 , 405, 906.

25. Rastogi, L.; Arunachalam, J.; Adv. Mater. Lett. 2013, 4, 548.

26. Nanda, J.; Sapra, S.; Sarma, D. D.; Chandrasekharan, N.; Hodes, G.; Chem. Mater. 2000, 12, 1018.

27. Rath, C.; Sahu, K. K.; Kulkarni, S. D.; Anand, S.; Date, S. K.; Das, R. P.; Mishra, N. C.; Appl. Phys. Lett. 1999, 75, 4171.

28. Corbierre, M. K.; Cameron, N. S.; Lennox, R. B.; Langmuir 2004, 20, 2867.

29. Manivannan, S.; Ramaraj, R.; Pure Appl. Chem. 2011, 83, 2041.

30. Wu, X.; Jiang, H.; Zheng, J.; Wang, X.; Gu, Z.; Chen, C.; J. Electroanal. Chem. 2011, 656, 174.

31. Kara, P.; Kerman, K.; Ozkan, D.; Meric, B.; Erdem, A.; Ozkan, Z.; Ozsoz, M.; Electrochem. Commun. 2002, 4, 705.

32. Issa, R.; Hamdan, N. A.; Noh, M. F. M.; Biotechnology 2010 , 9, 304.

33. Khezrian, S.; Salimi, A.; Teymourian, H.; Hallaj, R.; Biosens. Bioelectron. 2013, 43, 218.

34. Mao, K.; Wu, D.; Li, Y.; Ma, H.; Ni, Z.; Yu, H.; Luo, C.; Wei, Q.; Du, B.; Anal. Biochem. 2012, 422, 22.

Submitted: October 24, 2015 Published online: February 12, 2016 